\title{
Cosmological weak lensing
}

\author{
Yannick Mellier ${ }^{1,2}$, Ludovic van Waerbeke ${ }^{1,3}$, Francis Bernardeau ${ }^{4}$, and Ismael \\ Tereno $^{1,5}$ \\ 1 IAP, 98bis boulevard Arago, 75014 Paris, France \\ 2 Observatoire de Paris, LERMA, 61 avenue de l'Observatoire, 75014 Paris, France \\ 3 CITA, 60 St George Str., M5S 3H8, Toronto, Canada, \\ 4 SPhT, CE Saclay, 91191 Gif-sur-Yvette Cedex, \\ 5 Department of Physics, University of Lisbon 1749-016 Lisboa, Portugal.
}

\begin{abstract}
We present the current status of cosmic shear studies and their implications on cosmological models. Theoretical expectations and observational results are discussed in the framework of standard cosmology and CDM scenarios. The potentials of the next generation cosmic shear surveys are discussed.
\end{abstract}

\section{Introduction}

The gravitational deflection of light beams by large scale structures of the universe (cosmological lensing) amplifies and modifies the shape of distant galaxies and quasars. Magnification produces correlation between the density of foreground lenses and the apparent luminosity of distant galaxies or quasars (magnification bias), whereas distortion induces a correlation of ellipticity distribution of lensed galaxies (cosmic shear). In both cases, the properties of cosmological lensing signals probe the matter content and the geometry of universe and how perturbations grew and clustered during the past Gigayears.

Albeit difficult to detect, the recent cosmic shear detections claimed by several groups demonstrate that it is no longer a technical challenge. It is therefore possible to study the universe through a new window which directly probes dark matter instead of light and allows cosmologists to measure cosmological parameters and dark matter power spectrum from weak gravitational distortion.

\section{Weak cosmological lensing}

Let us assume that the shape of galaxies can be simply characterize by their surface brightness second moments $I(\boldsymbol{\theta})$, (see [1], [2] and references therein):

$$
M_{i j}=\frac{\int I(\boldsymbol{\theta}) \theta_{i} \theta_{j} \mathrm{~d}^{2} \theta}{\int I(\boldsymbol{\theta}) \mathrm{d}^{2} \theta} .
$$

Because of gravitational lensing, a galaxy with intrinsic ellipticity $\mathbf{e}$ is measured with an ellipticity $\mathbf{e}+\boldsymbol{\delta}$, where $\boldsymbol{\delta}$ is the gravitational distortion,

$$
\boldsymbol{\delta}=2 \gamma \frac{(1-\kappa)}{(1-\kappa)^{2}+|\gamma|^{2}}=\left(\delta_{1}: \frac{M_{11}-M_{22}}{\operatorname{Tr}(M)} ; \delta_{2}: \frac{2 M_{12}}{\operatorname{Tr}(M)}\right) .
$$


$\kappa$ and $\gamma$ are respectively the gravitational convergence and shear. Both depend on the second derivatives of the projected gravitational potential, $\varphi$ :

$$
\kappa(\boldsymbol{\theta})=\frac{1}{2}(\varphi, 11+\varphi, 22) ; \quad \gamma_{1}(\boldsymbol{\theta})=\frac{1}{2}(\varphi, 11-\varphi, 22) ; \quad \gamma_{2}(\boldsymbol{\theta})=\varphi, 12 .
$$

In the case of weak lensing, $\kappa<<1,|\gamma|<<1$ and $\delta \approx 2 \gamma$. Since large-scale structures have very low density contrast, this linear relation is in particular valid on cosmological scales.

Light propagation through an inhomogeneous universe accumulates weak lensing effects over Gigaparsec distances. Assuming structures formed from gravitational growth of Gaussian fluctuations, cosmological weak lensing can be predicted from Perturbation Theory at large scale. To first order, the convergence $\kappa(\boldsymbol{\theta})$ at angular position $\boldsymbol{\theta}$ is given by the line-of-sight integral

$$
\kappa(\boldsymbol{\theta})=\frac{3}{2} \Omega_{0} \int_{0}^{z_{s}} n\left(z_{s}\right) \mathrm{d} z_{s} \int_{0}^{\chi(s)} \frac{D\left(z, z_{s}\right) D(z)}{D\left(z_{s}\right)} \delta(\chi, \boldsymbol{\theta})[1+z(\chi)] \mathrm{d} \chi
$$

where $\chi(z)$ is the radial distance out to redshift $z, D$ the angular diameter distances, $n\left(z_{s}\right)$ is the redshift distribution of the sources. $\delta$ is the mass density contrast responsible for the deflection at redshift $z$. Its amplitude at a given redshift depends on the properties of the power spectrum and its evolution with look-back-time.

The cumulative weak lensing effects of structures induce a shear field which is primarily related to the power spectrum of the projected mass density, $P_{\kappa}$. Its statistical properties can be recovered by the shear top-hat variance [17,8,19,

$$
\left\langle\gamma^{2}\right\rangle=\frac{2}{\pi \theta_{c}^{2}} \int_{0}^{\infty} \frac{\mathrm{d} k}{k} P_{\kappa}(k)\left[J_{1}\left(k \theta_{c}\right)\right]^{2},
$$

the aperture mass variance 21,35

$$
\left\langle M_{\mathrm{ap}}^{2}\right\rangle=\frac{288}{\pi \theta_{c}^{4}} \int_{0}^{\infty} \frac{\mathrm{d} k}{k^{3}} P_{\kappa}(k)\left[J_{4}\left(k \theta_{c}\right)\right]^{2},
$$

and the shear correlation function $17,8,19$ :

$$
\langle\gamma \gamma\rangle_{\theta}=\frac{1}{2 \pi} \int_{0}^{\infty} \mathrm{d} k k P_{\kappa}(k) J_{0}(k \theta),
$$

where $J_{n}$ is the Bessel function of the first kind. Higher order statistics, like the skewness of the convergence, $s_{3}(\theta)$, can also be computed. They probe non Gaussian features in the projected mass density field, like massive clusters or compact groups of galaxies. (see [3]; 传 and references therein). The amplitude of cosmic shear signal and its sensitivity to cosmology can be illustrated in the fiducial case of a power law mass power spectrum with no cosmological constant and a background population at a single redshift $z$. In that case $\left\langle\kappa(\theta)^{2}>\right.$ and $s_{3}(\theta)$ write:

$$
<\kappa(\theta)^{2}>^{1 / 2}=<\gamma(\theta)^{2}>^{1 / 2} \approx 1 \% \sigma_{8} \Omega_{m}^{0.75} z_{s}^{0.8}\left(\frac{\theta}{1^{\prime}}\right)^{-\left(\frac{n+2}{2}\right)},
$$


and

$$
s_{3}(\theta)=\frac{\left\langle\kappa^{3}\right\rangle}{\left\langle\kappa^{2}\right\rangle^{2}} \approx 40 \Omega_{m}^{-0.8} z_{s}^{-1.35},
$$

where $n$ is the spectral index of the power spectrum of density fluctuations. Therefore, in principle the degeneracy between $\Omega_{m}$ and $\sigma_{8}$ can be broken when both the variance and the skewness of the convergence are measured.

\section{Detection of weak distortion signal}

\subsection{Observational challenge}

Eq. (8) shows that the amplitude of weak lensing signal is of the order of few percents, which is much smaller than the intrinsic dispersion of ellipticity distribution of galaxies. van Waerbeke et al (河) explored which strategy would be best suited to probe statistical properties of such a small signal. They have shown that the variance of $\kappa$ can be measured with a survey covering about 1 $\mathrm{deg}^{2}$, whereas for the skewness one needs at least $10 \mathrm{deg}^{2}$. Furthermore, more than $100 \mathrm{deg}^{2}$ must be observed in order to uncover information on $\Omega_{\Lambda}$ or the shape of the power spectrum over scales larger than 1 degree. For $\Omega_{m}=0.3$ and $\sigma_{8}=1$, the limiting shear amplitude can be simply expressed as follows

$$
<\gamma(\theta)^{2}>^{1 / 2}=0.3 \%\left[\frac{A_{T}}{100 \operatorname{deg}^{2}}\right]^{\frac{1}{4}} \times\left[\frac{\sigma_{\epsilon_{g a l}}}{0.4}\right] \times\left[\frac{n}{20}\right]^{-\frac{1}{2}} \times\left[\frac{\theta}{10^{\prime}}\right]^{-\frac{1}{2}},
$$

where $A_{T}$ is the total sky coverage of the survey. The numbers given in the brackets correspond to a measurement at $3-\sigma$ confidence level of the shear variance. Eq. (10) contains the specifications of a cosmic shear survey.

\subsection{First detection of weak distortion}

Despite technical limitations discussed above, on scale significantly smaller than one degree, non-linear structures dominate and increase the amplitude of the lensing signal, making its measurement easier. Few teams started such surveys during the past years and succeeded to get a significant signal. Table 1 lists some published results. Since each group used different telescopes and adopted different observing strategy and data analysis techniques, one can figure out the reliability of the final results. Figure 1 show that all these independent results are in very good agreement? This is a convincing demonstration that the expected correlation of ellipticities is real.

1 [26] data are missing because depth is different so the sources are at lower redshift and the amplitude of the shear is not directly comparable to other data plotted 
Table 1. Present status of cosmic shear surveys with published results.

\begin{tabular}{|c|c|c|c|c|}
\hline Telescope & Pointings & Total Area & Lim. Mag. & Ref.. \\
\hline$\overline{\mathrm{CFHT}}$ & $5 \times 30^{\prime} \times 30^{\prime}$ & $1.7 \mathrm{deg}^{2}$ & $\mathrm{I}=24$. & [7] [vWME+] \\
\hline CTIO & $3 \times 40^{\prime} \times 40^{\prime}$ & $1.5 \mathrm{deg}^{2}$ & $\mathrm{R}=26$ & [9][WTK+] \\
\hline WHT & $14 \times 8^{\prime} \times 15^{\prime}$ & $0.5 \mathrm{deg}^{2}$ & $\mathrm{R}=24$. & 10] [BRE] \\
\hline CFHT & $6 \times 30^{\prime} \times 30^{\prime}$ & $1.0 \mathrm{deg}^{2}$ & $\mathrm{I}=24$. & 12 [KWL] \\
\hline VLT/UT1 & $50 \times 7^{\prime} \times 7^{\prime}$ & $0.6 \mathrm{deg}^{2}$ & $\mathrm{I}=24$. & 13] $[\mathrm{MvWM}+]$ \\
\hline HST/WFPC2 & $1 \times 4^{\prime} \times 42^{\prime}$ & $0.05 \mathrm{deg}^{2}$ & $\mathrm{I}=27$. & 14 \\
\hline CFHT & $4 \times 120^{\prime} \times 120^{\prime}$ & $6.5 \mathrm{deg}^{2}$ & $\mathrm{I}=24$. & $15][\mathrm{vWMR}+]$ \\
\hline HST/STIS & $121 \times 1^{\prime} \times 1^{\prime}$ & $0.05 \mathrm{deg}^{2}$ & $\mathrm{~V} \approx 26$ & 25 \\
\hline CFHT & $5 \times 126^{\prime} \times 140^{\prime}$ & 24. $\operatorname{deg}^{2}$ & $\mathrm{R}=23.5$ & 26 \\
\hline CFHT & $10 \times 126^{\prime} \times 140^{\prime}$ & 53. $\operatorname{deg}^{2}$ & $\mathrm{R}=23.5$ & 27 \\
\hline CFHT & $4 \times 120^{\prime} \times 120^{\prime}$ & $8.5 \mathrm{deg}^{2}$ & $\mathrm{I}=24$. & 28 \\
\hline HST/WFPC2 & $271 \times 2.1 \times 2.1$ & $0.36 \mathrm{deg}^{2}$ & $\mathrm{I}=23.5$ & 23 \\
\hline Keck+WHT & $\begin{array}{c}173 \times 2^{\prime} \times 8^{\prime} \\
+13 \times 16^{\prime} \times 8^{\prime} \\
7 \times 16^{\prime} \times 16^{\prime}\end{array}$ & $1.6 \mathrm{deg}^{2}$ & $\mathrm{R}=25$ & 16 \\
\hline
\end{tabular}

\subsection{Nature of the weak distortion signal}

The detection of coherent signal is not a demonstration of its very nature. Even if a cosmological signal were expected, it could be contaminated by systematics, like optical and atmospheric distortions, which mix together with the gravitational shear. Contrary to lensing effects, these contributions are visible also on stars and can be corrected (using for example the KSB method, 20]). However, stars often show strong anisotropic shape with elongation much larger than the expected amplitude of the gravitational distortion. The reliability of artificial anisotropy corrections is therefore a critical step of the weak lensing analysis (see for example [6], [7] [10] and [11]). An elegant way to check whether corrections are correctly done and to confirm the gravitational nature of the signal is to decompose the signal into E- and B- modes. The E-mode contains signal produced by gravity-induced distortion whereas the B-mode is a pure curl-component, so it only contains intrinsic ellipticity correlation or systematics residuals. Both modes have been extracted using the aperture mass statistics by van Waerbeke et al ([15], 24]) and Pen et al ([28]) in the VIRMOS-DESCART survey as well as by Hoekstra et al (27) in the Red Cluster Sequence survey. In both samples, the E-mode dominates the signal, although a small residual is detected in the B-mode. This strongly supports the gravitational origin of the distortion.

An alternative to gravitational lensing effect could be an intrinsic correlations of ellipticities of galaxies produced by proximity effects. It could results from galaxy formation processes. Several recent numerical and theoretical studies (see for example [30]; 31]) show that intrinsic correlations are negligible on scales beyond one arc-minute, provided that the survey is deep enough. In that case, most lensed galaxies along a line of sight are spread over Gigaparsec scales and have no physical relation with its apparent neighbors. Hence, since most cosmic survey 


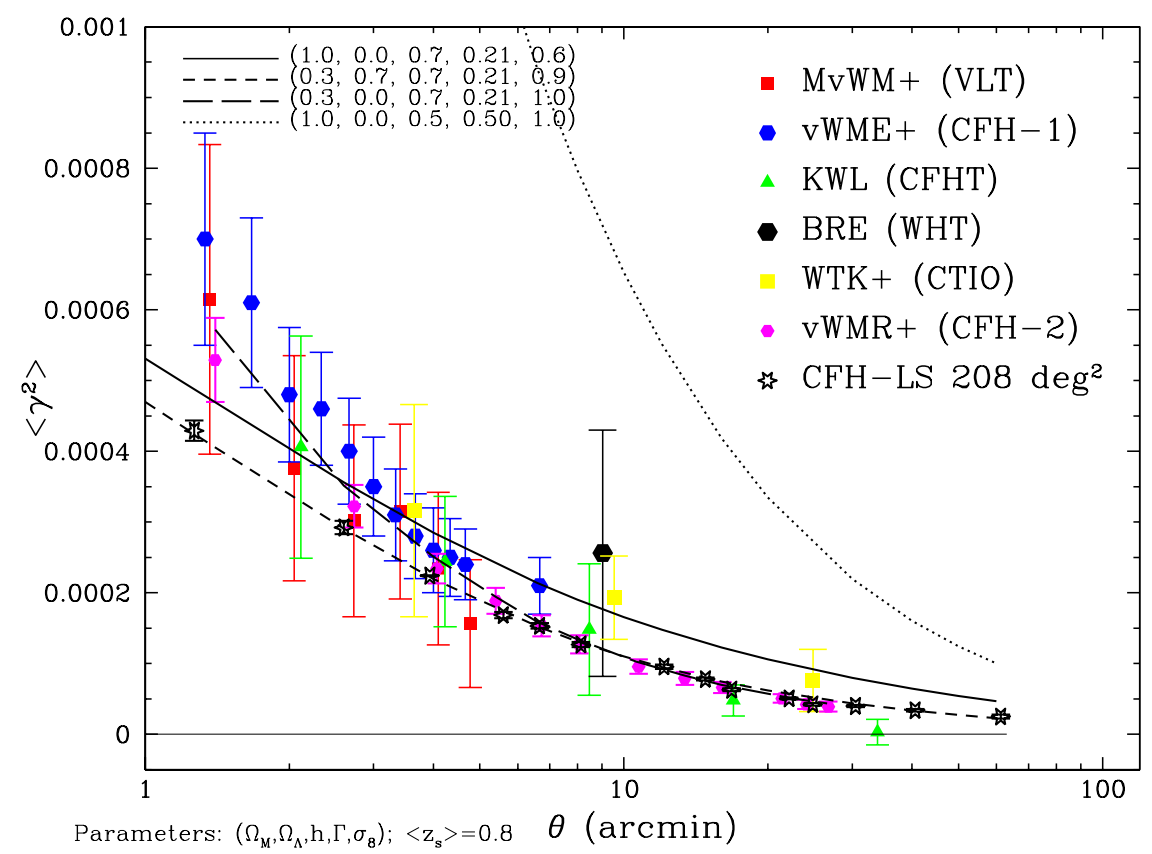

Fig. 1. Top hat variance of shear as function of angular scale from 6 cosmic shear surveys. The CFHT-LS (open black stars) illustrates the expected signal from a large survey covering $200 \mathrm{deg}^{2}$. For most points the errors are smaller than the stars.

are deep, they are almost free of intrinsic correlations. We therefore are confident that the signal measured by all teams is a genuine cosmic shear signal.

\section{Cosmological interpretations}

\subsection{2-point statistics and variance}

A comparison of the top-hat variance of shear with some realistic cosmological models is ploted in Figure 1. The amplitude of the shear has been scaled using photo- $z$ which gives $\langle z\rangle \approx 1$. On this plot, we see that standard COBEnormalized CDM is ruled at a $10-\sigma$ confidence level. However, the degeneracy between $\Omega_{m}$ and $\sigma_{8}$ discussed in the previous section still hampers a strong discrimination among most popular cosmological models. The present-day constraints resulting from independent analyses by Maoli et al ([13), Rhodes et al ([14), van Waerbeke et al (15,24]), Hoekstra et al (26,27) and Réfrégier et al (23]) can be summarized by the following conservative boundaries (90\% confidence level):

$$
0.05 \leq \Omega_{m} \leq 0.8 \quad \text { and } \quad 0.5 \leq \sigma_{8} \leq 1.2,
$$




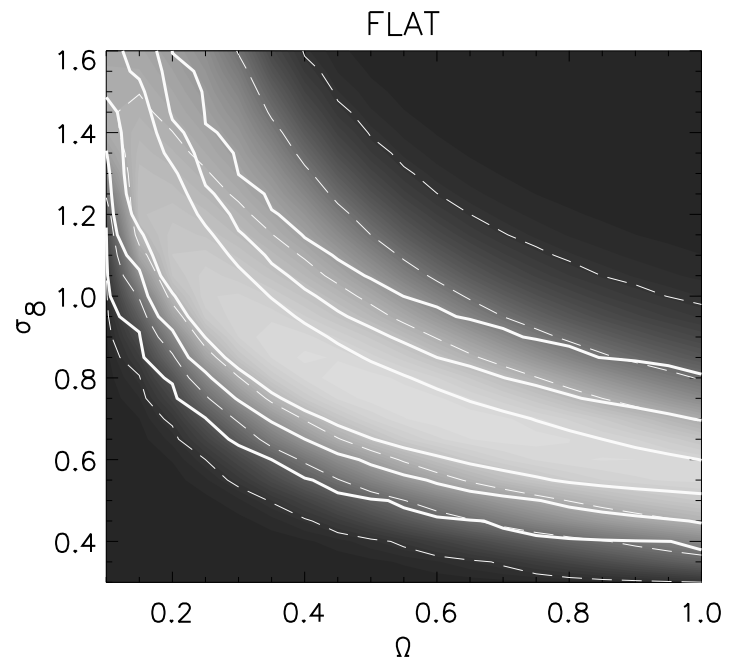

Fig. 2. Constraints on $\Omega$ and $\sigma_{8}$ for the flat cosmologies. The confidence levels are $[68,95,99.9]$ from the brightest to the darkest area. The gray area and the dashed contours correspond to the computations with a full marginalisation over the default prior $\Gamma \in[0.05,0.7]$ and $z_{s} \in[0.24,0.64]$. The thick solid line contours are obtained from the prior $\Gamma \in[0.1,0.4]$ and $z_{s} \in[0.39,0.54]$ (which is a mean redshift $\bar{z}_{s} \in[0.8,1.1]$ ). From van Waerbeke et al. (2002).

and, in the case of a flat-universe with $\Omega_{m}=0.3$, they lead to $\sigma_{8} \approx 0.9$.

\subsection{The 3-point shear correlations function: breaking the $\Omega_{\mathrm{m}}-\sigma_{8}$ degeneracy}

The measurement of non-Gaussian features needs informations on higher order statistics than variance. Although the afore mentioned skewness of $\kappa$ looks a promising quantity for this purpose, its measurements suffers from a number a practical difficulties which are not yet fixed. Recently, Bernardeau, van Waerbeke \& Mellier ([37) have proposed an alternative method using some specific patterns in the shear three-point function. Despite the complicated shape of the three-point correlation pattern, they uncovered it can be used for the measurement of non-Gaussian features. Their detection strategy based on their method has been tested on ray tracing simulations and turns out to be robust, usable in patchy catalogs, and quite insensitive to the topology of the survey.

Bernardeau, Mellier \& van Waerbeke (38]) used the analysis of the 3-point correlations function on the VIRMOS-DESCART data. Their results on Figure 3 show a $2.4 \sigma$ signal over four independent angular bins, or equivalently, a $4.9-\sigma$ confidence level detection with respect to measurements errors on scale of about 2 to 4 arc-minutes. The amplitude and the shape of the signal are consistent with theoretical expectations obtained from ray-tracing simulations. This result 


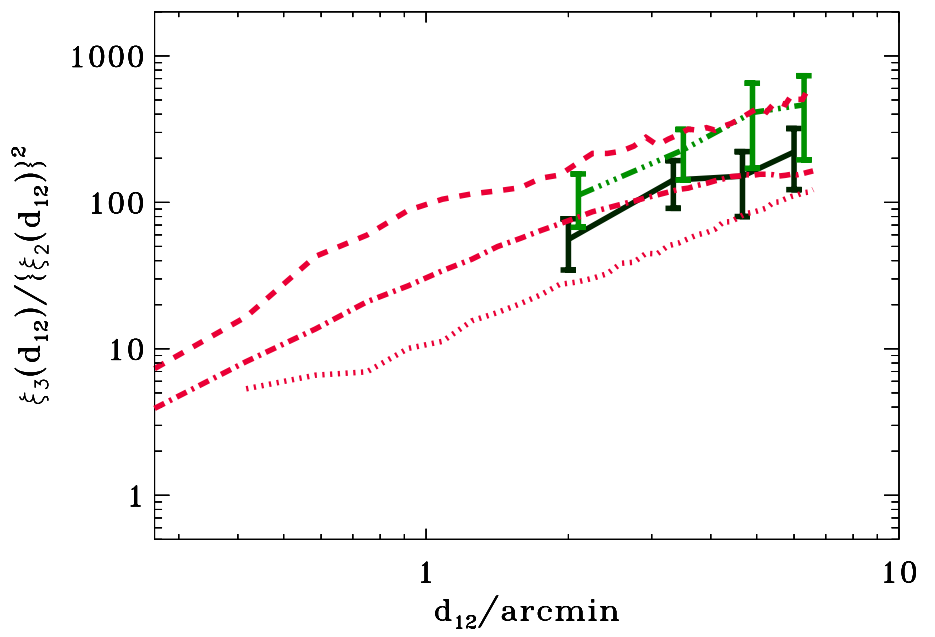

Fig. 3. Results for the VIRMOS-DESCART survey of the reduced three point correlation function (338). The solid line with error bars shows the raw results, when both the $E$ and $B$ contributions to the two-point correlation functions are included. The dot-dashed line with error bars corresponds to measurements where the contribution of the $B$ mode has been subtracted out from the two-point correlation function. These measurements are compared to results obtained in $\tau \mathrm{CDM}, \mathrm{OCDM}$ and $\Lambda \mathrm{CDM}$ simulations (dashed, dotted and dot-dashed lines respectively).

supports the idea that the measure corresponds to a cosmological signal due to the gravitational instability dynamics. Moreover, its properties could be used to put constraints on the cosmological parameters, in particular on the density parameter of the Universe. Although the errors are still large to permit secure conclusions, one clearly see that the amplitude and the shape of the 3-point correlations function match the most likely cosmological models. Remarkably, the $\Lambda \mathrm{CDM}$ scenario perfectly fit the data points.

The Bernardeau et al. ([38]) result is the first detection of non-Gaussian features in a cosmic shear survey and it opens the route to break the $\Omega_{\mathrm{m}}-\sigma_{8}$ degeneracy. Furthermore, this method is weakly dependent on other parameters, like the cosmological constant or the properties of the power spectrum. However, there are still some caveats which may be considered seriously. One difficulty is the source clustering which could significantly perturb high-order statistics (Hamana et al 2000, 32 ). If so, multi-lens plane cosmic shear analysis will be necessary which implies a good knowledge of the redshift distribution. For very deep cosmic shear surveys, this could be could be a challenging issue. 


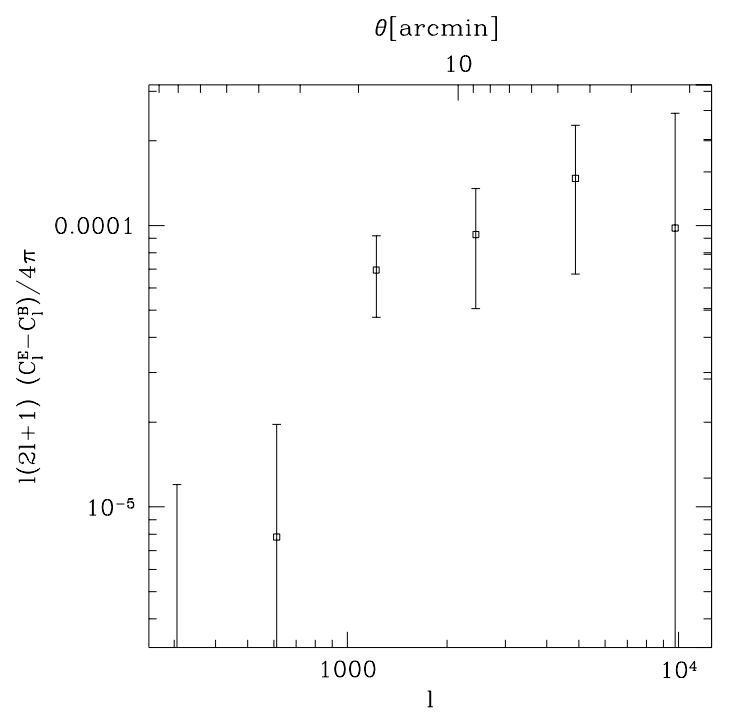

Fig. 4. Cosmological results from cosmic shear surveys: The angular power spectrum of the convergence field from the VIRMOS-DESCART survey is plotted (From Pen et al 2001). These are the first $C(l)$ of dark matter ever measured in cosmology.

\section{$5 \quad$ What next?}

Because on going surveys increase both in solid angle and in number of galaxies, they will quickly improve the accuracy of cosmic shear measurements, at a level where $\Omega_{m}$ and $\sigma_{8}$ will be known with a $10 \%$ accuracy. Since it is based on gravitational deflection by intervening matter spread over cosmological scales, the shape of the distortion field also probes directly the shape of the projected power spectrum of the (dark) matter. Pen et al (28]) already explored its properties measuring for the first time the $C(l)$ of the dark matter (see Figure (4). We therefore know this is feasible with present data. However, we expect much more within the next decade. Surveys covering hundreds of degrees, with multi-bands data in order to get redshift of sources and possibly detailed information of their clustering properties, are scheduled. The CFHT Legacy Survey ${ }^{2}$ will cover 200 $\operatorname{deg}^{2}$ and is one of those next-generation cosmic shear survey. Figures 1 and a shows it potential for cosmology. On figure 1 we simulated the expected signal to noise of the shear variance as function of angular scale for a $\Lambda \mathrm{CDM}$ cosmology. The error bars are considerably reduced as compared to present-day survey. On Fig. 5, we compare the expected signal to noise ratio of the CFHT Legacy Survey with the expected amplitude of the angular power spectrum for several theoretical quintessence fields models. It shows that even with $200 \mathrm{deg}^{2}$ which include multi-color informations in order to get redshift of sources, one can al-

$\overline{2}$ http://www.cfht.hawaii.edu/Science/CFHLS/ 


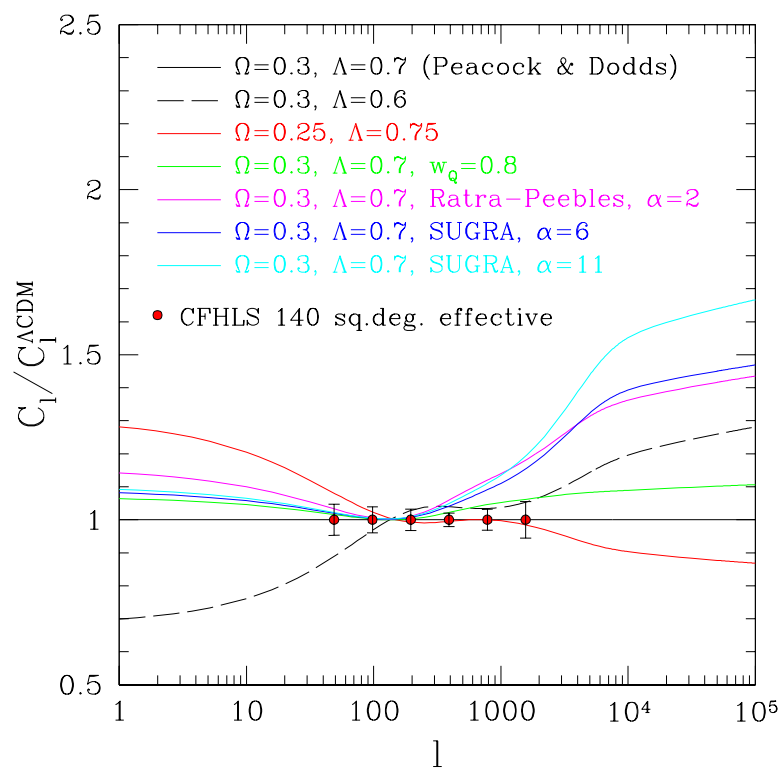

Fig. 5. The future of from cosmic shear surveys: Theoretical expectations of the CFHT Legacy Survey. The dots with error bars are the expected measurements of cosmic shear data from the $208 \mathrm{deg}^{2}$ of the CFHT Legacy Survey. The lines shows various models discussed by Benabed \& Bernardeau ([39).

ready obtain interesting constraints on cosmology beyond standard models. The use of cosmic shear data can be much more efficient if they are used together with other surveys, like CMB (Boomerang, MAP, Planck), SNIa surveys, or even galaxy surveys (2dF, SDSS). For example, SDSS will soon provide the 100, 000 quasars with redshifts. Ménard \& Bartelmann (40]) have recently explored the interest of this survey in order to cross-correlate the foreground galaxy distribution with the quasar population. The expected magnification bias generated by dark matter associated with foreground structures as mapped by galaxies depends on $\Omega_{m}$ and the biasing $\sigma_{8}$. In principle magnification bias in the SDSS quasar sample can provide similar constrains as cosmic shear.

\section{Aknowledgements}

We thank M. Bartelmann, K. Benabed, D. Bond, T. Hamana, H. Hoekstra, B. Ménard, S. Prunet and P. Schneider for useful discussions. This work was supported by the TMR Network "Gravitational Lensing: New Constraints on Cosmology and the Distribution of Dark Matter" of the EC under contract No. ERBFMRX-CT97-0172. YM thanks the organizers of the meeting for financial support. 


\section{References}

1. Mellier, Y.; 1999 ARAA 37, 127.

2. Bartelmann, M.; Schneider, P.; 2001 Phys. Rep. 340, 292.

3. Bernardeau, F.; van Waerbeke, L.; Mellier, Y.; 1997 A\&A 322, 1.

4. Jain, B.; Seljak, U.; 1997 ApJ 484, 560.

5. van Waerbeke, L.; Bernardeau, F.; Mellier, Y.; 1999 A\&A 342, 15.

6. Erben, T.; van Waerbeke, L.; Bertin, E.; Mellier, Y.; Schneider, P.; 2001 A\&A 366,717 .

7. van Waerbeke, L.; Mellier, Y.; Erben, T.; et al.; 2000 A\&A 358, 30 [vWME+].

8. R. Blandford, A. Saust, T. Brainerd, J. Villumsen; 1991 MNRAS 251, 600

9. Wittman, D.; Tyson, J.A.; Kirkman, D.; Dell'Antonio, I.; Bernstein, G. 2000a Nature 405, 143 [WTK+].

10. Bacon, D.; Réfrégier; A., Ellis, R.S.; 2000 MNRAS 318, 625 [BRE].

11. Bacon, D.; Réfrégier; A., Clowe, D., Ellis, R.S.; 2000 MNRAS 325, 1065.

12. Kaiser, N.; Wilson, G.;, Luppino, G. 2000 preprint, astro-ph/0003338 [KWL].

13. Maoli, R.; van Waerbeke, L.; Mellier, Y.; et al.; 2001 A\&A 368, 766 [MvWM+].

14. Rhodes, J.; Réfrégier, A., Groth, E.J.; 2001 ApJ 536, 79.

15. van Waerbeke, L.; Mellier, Y.; Radovich, M.; et al.; 2001 A\&A 374, 757 [vWMR+].

16. Bacon, D., Massey, R., , Réfrégier, A.,. Ellis, R. astro-ph/0203134

17. J. Miralda-Escudé; 1991 ApJ380,1

18. Pen, U-L., Van Waerbeke, L., Mellier, Y.; 2002 ApJ 567, 31

19. Kaiser, N. 1992 ApJ 388, 272

20. kaiser, N., Squires, G., Broadhurst, T. 1995, ApJ 449, 460.

21. Kaiser, N. et al., 1994, in Durret et al., Clusters of Galaxies, Eds Frontières

22. P. Schneider, L. Van Waerbeke, B. Jain, G. Kruse; 1998 ApJ333, 767.

23. A. Réfrégier, J. Rhodes, E. Groth, ApJL, in press, astro-ph/0203131

24. L. Van Waerbeke, Y. Mellier, R. Pello et al., A\& A, in press, astro-ph/0202503

25. Hämmerle, H.; Miralles, J.-M.; Schneider, P.; Erben, T.; Fosbury, R.A.E.; Freudling, W.; Pirzkal; N., Jain, B.; White, S.D.M.; 2002 A\&A385 , 743

26. Hoekstra, H.; Yee, H.; Gladders, M.D. 2001 ApJ 558, L11

27. H. Hoekstra, H. Yee, M. Gladders, ApJ, in press, astro-ph/0204295

28. Pen, U.; van Waerbeke, L.; Mellier, Y.; 2001 ApJ in press astro-ph/0109182.

29. Pierpaoli, E., Scott, D., White, M. 2001 MNRAS 325, 77.

30. Crittenden, R.G.; Natarajan, P.; Pen, U.; Theuns, T. 2001 ApJ 559, 552.

31. Mackey, J.; White, M.; Kamionkowski, M.; 2001 preprint, astro-ph/0106364.

32. Hamana, T. et al. 2000. preprint astro-ph/0012200

33. Davis, M.; Newman, J.; Faber, S.; Phillips, A.; 2000 Proc. ESO/ECF/ESA on Deep Fields Springer.

34. Le Fèvre, O.; Saisse, M.; Mancini, M.; 2000 SPIE 4008, 546.

35. Schneider, P. 1998 ApJ 498, 43.

36. van Waerbeke, L. 1998 A\&A 334, 1.

37. Bernardeau, F., van Waerbeke, L., Mellier, Y. 2002 preprint astro-ph/0201029.

38. Bernardeau, F., Mellier, Y., van Waerbeke, L. 2002 A\&A in press. preprint astro$\mathrm{ph} / 0201032$

39. Benabed, K.; Bernardeau, F.; 2001 preprint, astro-ph/0104371.

40. Ménard, B., Bartelmann, M. 2002 preprint astro-ph/0203163 